\section{Metabolic changes in the kidney}

Salt-induced hypertension and its associated renal and cardiovascular complications are a major cause of chronic kidney disease (CKD), but the mechanisms are not well understood. A new study by Markus Rinschen, Oleg Palygin, Alexander Staruschenko, Gary Siuzdak and colleagues reveals the contributions of metabolic changes in renal glomeruli in a rat model of kidney hypertension and suggests that metabolic interventions might be useful in treating hypertension-induced kidney disease.

"Understanding the functional processes in the whole kidney presents a tremendous experimental challenge owing to the structural and functional heterogeneity of kidney tissue, and the rapid pathophysiological changes that occur during the progression of disease states like hypertension," explains Palygin. "In order to directly assess the metabolic aspects of hypertension-induced glomerular sclerosis we used a multi-omics strategy, integrating metabolomics, phosphoproteomics and proteomics."

The researchers performed untargeted and targeted metabolomic analyses in a well-known model of kidney hypertension, the Dahl salt-sensitive (DSS) rat. They found increases in lipid breakdown and reductions in branched-chain amino acids in DSS rats. Oxidative stress and reductions in products of central carbon metabolism were also found in glomeruli.

Phosphoproteomic analysis on glomeruli of the DSS rat revealed the activation of AMPK and the inhibition of the kinase-containing complex mTORC1, and suggested that metabolic signalling through these kinases controls major pathophysiological pathways in glomeruli.

Using proteomic analysis, the researchers showed that alterations in the abundance of metabolic enzymes correlated with the metabolic changes seen during early stages of hypertensive kidney disease.

The researchers say that their findings indicate that metabolic or dietary interventions might be useful in preventing the adverse effects of hypertension on the kidney.

Rebecca Kelsey

ORIGINAL ARTICLE Rinschen, M. M. et al. Metabolic rewiring of the hypertensive kidney. Sci. Signal. 12, eaax9760 (2019)

\title{
IMMUNOLOGY
}

\section{Macrophages react to remote challenges}

Systemic inflammation is typically observed in response to sepsis, but myocardial infarction (MI) and stroke can also have systemic immune effects, including the induction of emergency haematopoiesis. A new study shows that "macrophages everywhere in the body know when danger is afoot, even when the danger is distant," explains Matthias Nahrendorf.

"We wondered if macrophages react to remote inflammation, how they might sense it and whether it matters to the host," explains Nahrendorf. The researchers then challenged mice with MI, stroke or sepsis and examined subsequent changes in macrophage populations in the kidney, heart, liver and brain. "I was most surprised by the reduction in macrophages numbers, even in the kidney, shortly after the onset of bacteraemia," notes Nahrendorf. "We looked at this carefully in the heart and found that macrophages take up circulating bacteria and then die." After this initial sepsis-induced reduction, macrophages in the kidney cortex and medulla expanded and remained above normal levels for over 2 months, owing to both resident cell proliferation and recruitment of circulating cells. Injury, in particular sepsis, altered the gene expression profile of kidney macrophages, which was most similar to that of heart macrophages.

The researchers also noted that lung macrophages responded to MI with a strong upregulation of proliferation genes; this change was not seen after stroke or sepsis. "Unexpectedly, mice survived pneumonia better if they had a prior MI," remarks Nahrendorf. "Lung macrophages seem to acquire a different phenotype after MI we sometimes call it 'bracing' - that better prepares them for subsequent challenges," explains Nahrendorf.

"We need to assess how long these changes in macrophage phenotypes persist, and we will follow up on differentially expressed genes," adds Nahrendorf. "It would also be worthwhile to test whether chronic kidney disease induces changes in tissue macrophages throughout the body."

Monica Wang

ORIGINAL ARTICLE Hoyer, F. F. et al. Tissue-specific

macrophage responses to remote injury impact the outcome of subsequent local immune challenge. Immunity 51, 899-914 (2019)

\section{NEPHROTOXICITY}

\section{Toxins linked to chronic interstitial nephritis in agricultural communities}

Chronic interstitial nephritis in agricultural communities (CINAC) is a disease that primarily affects young agricultural workers from low-income, tropical countries. Although heat stress has been proposed to have a role, the aetiology of this disease has not been determined. New findings suggest that CINAC is a toxin-induced nephropathy that affects the calcineurin signalling pathway. "We found a specific lesion in the kidneys of patients with CINAC that resembled lesions found in kidneys of transplant recipients treated with calcineurin inhibitors and of patients exposed to other nephrotoxic drugs that affect calcineurin signalling," explains Marc de Broe. "Although we do not yet have proof, we suspect that pesticides, used in agriculture, are responsible for this nephropathy."

To better understand the aetiology of CINAC, Benjamin Vervaet, de Broe and colleagues examined renal biopsy samples from patients with CINAC. "We started with clearly established cases of CINAC from
Sri Lanka, El Salvador and India but also examined biopsy samples from patients in regions of France where the use of pesticides is well documented," says de Broe. They found specific lesions in the proximal tubules, characterized by an accumulation of enlarged, dysmorphic lysosomes with dispersed aggregates together with a tubular atrophy, loss of tubular cell proliferation and interstitial fibrosis. Identification of the same lesions in patients treated with calcineurin inhibitors and other nephrotoxic agents, and in the kidneys of rats treated with ciclosporin, led the researchers to propose that CINAC is a toxin-induced nephropathy caused by factors that affect calcineurin signalling. De Broe and colleagues now hope to identify the responsible toxins, with the aim of introducing measures to prevent the development of CINAC in agricultural communities.

Susan J. Allison

ORIGINAL ARTICLE Vervaet, B. A. et al. Chronic interstitial nephritis in agricultural communities is a toxin induced proximal tubular nephropathy. Kidney Int. https://doi.org/ 10.1016/j.kint.2019.11.009 (2019) 